\title{
High yielding synthesis of dehydroamino acid and dehydropeptide derivatives
}

\author{
Paula M. T. Ferreira, Hernâni L. S. Maia, Luís S. Monteiro and Joana Sacramento \\ Department of Chemistry, University of Minho, Gualtar, P-4700-320 Braga, Portugal
}

Received (in Cambridge, UK) 14th June 1999, Accepted 19th October 1999

By using a 4-dimethylaminopyridine (DMAP) catalysed reaction of $\beta$-hydroxyamino acid derivatives with tertbutyl pyrocarbonate $\left[(\mathrm{Boc})_{2} \mathrm{O}\right]$, dehydroamino acid derivatives are obtained in high yields. The same methodology applied to dipeptides with a $\beta$-hydroxyamino acid residue gives the corresponding dipeptides with a dehydroamino acid residue.

\section{Introduction}

Nisin, a well known preservative in the food industry, epidermin, a therapeutic agent against acne, and ancovenin, an immunologically active compound, are examples of the many different compounds belonging to the important class of natural bacteriocins known as lantibiotics. Owing to the wide variety of biological activities and applications found among the known compounds and also to the economical importance of many of them, lantibiotics offer a promising field for exploration of new compounds with new biological properties and new applications. Lantibiotics are polycyclic peptides, which, in addition to lanthionine, contain $\alpha, \beta$-dehydroamino acid residues. The latter have been found also in the molecules of several enzymes from plant and bacterial sources and have been used as linkers in solid phase peptide synthesis and in synthetic peptides aimed at structure-activity relationship studies, since they affect both chemical reactivity and conformation. ${ }^{1-4}$ Another important application for dehydroamino acid derivatives is their use in addition reactions yielding new amino acids as the corresponding $\beta$-substituted products. ${ }^{5,6} \mathrm{~A}$ key step to progress in this field is, thus, the production of $\alpha, \beta$-didehydroamino acid derivatives suitable for incorporation into peptide sequences or, otherwise, a method for dehydration of appropriate precursors. An obvious approach to these compounds is the dehydration of $\beta$-hydroxyamino acid derivatives as found in the biosynthetic route to lantibiotics in which their precursors containing serine and threonine residues are dehydrated to give the corresponding dehydroalanine $(\Delta$ Ala $)$ and dehydroaminobutyric acid $(\triangle \mathrm{Abu})$ derivatives. In fact, this has been the most widely used approach to the chemical synthesis of dehydroamino acid derivatives, mainly for the case of dehydroalanine and dehydroaminobutyric acid..$^{4,7-9}$ Several other methods have been developed for the synthesis of these compounds, which include Hofmann degradation of $\alpha, \beta$-diaminopropionyl residues, ${ }^{10}$ reduction of azidoacrylates, ${ }^{3}$ hydrolysis of unsaturated oxazolinones ${ }^{11}$ and condensation of $\alpha$-ketoacids with amides or nitriles. ${ }^{12,13}$ As described in the literature, all the above methods are usually low yielding, multistep processes requiring tedious purifications to remove side products; this may be related partially to the fact that, together with some of their derivatives, they undergo polymerisation and hydrolysis fairly easily. In the case of dehydroaminobutyric acid derivatives the work-up procedures are complicated by the formation of two stereoisomers. Thus, there is still a need for developing a simple and efficient approach to these compounds and in this paper we report such an approach; it is again based on $\beta$-elimination from $\beta$-hydroxyamino acid derivatives but the reactions are almost quantitative and no mixtures of isomers have been found, so far. In addi- tion, this method is equally suitable for the ready dehydration of peptide derivatives containing one or more $\beta$-hydroxyamino acid residues.

\section{Results and discussion}

Our approach was initially based on Berkowitz and Pederson's method for simultaneous amine and carboxy protection of amino acids with benzyl chloroformate in the presence of DMAP and triethylamine. ${ }^{14}$ We found that under these conditions serine undergoes elimination, the only product isolated being the corresponding fully protected dehydroalanine derivative (Z- $\triangle$ Ala-OBn) in a yield of $51 \%{ }^{15}$ Applying the same procedure to several amino acids protected either at their $\mathrm{N}$-terminus or at both the $\mathrm{N}$ - and the C-terminus, the yields obtained were within the range 56-76\%. With a threonine derivative, although all the starting material was consumed, we failed to obtain any pure product and NMR spectroscopy of the reaction mixture was consistent with the presence of the two isomers of dehydroaminobutyric acid.

The experimental conditions usually required to cleave most of the protecting groups we used for N-protection, viz. benzyloxycarbonyl $(\mathrm{Z}), p$-nitrobenzyloxycarbonyl $\left[\mathrm{Z}\left(\mathrm{NO}_{2}\right)\right]$ and tosyl (Tos) are possibly too drastic to be applied to dehydroamino acid derivatives. However, their use was intended to allow the investigation of their cleavage from dehydroamino acids by mild electrolysis according to techniques developed earlier by our team. ${ }^{16,17}$ In these studies we showed that electrolysis is facilitated when two acyl groups are attached to the nitrogen atom. We also showed that tert-butoxycarbonyl (Boc) not only fulfils efficiently the role required for the second acyl group but it is easy to introduce by reaction of the previously protected material with tert-butyl pyrocarbonate, $(\mathrm{Boc})_{2} \mathrm{O}$, in the presence of DMAP as catalyst, according to Ragnarsson's method for tert-butoxylation of amides. ${ }^{18}$ This acylation with Boc prior to electrolysis resembles significantly the method we had taken advantage of to dehydrate serine. Thus, the use of two equivalents of tert-butyl pyrocarbonate would suit both tasks, i.e. further acylation for later selective deprotection by electrolysis, if at all required, and dehydration (2, Scheme 1). Although the increased bulk thus created at the nitrogen atom was expected to assist elimination during the dehydration step, the results obtained exceeded our expectations; in fact, the only product isolated was the corresponding dehydrated diacyl ester in an almost quantitative yield. ${ }^{19}$ Moreover, with both threonine and $\beta$-hydroxyphenylalanine derivatives (threo type) the reaction was stereoselective, giving only the $Z$-isomer as shown by NMR spectroscopy. This selectivity seems to result again from the 
Table 1 Results obtained in the synthesis of dehydroamino acid derivatives

\begin{tabular}{|c|c|c|}
\hline Reagent & Product & $\begin{array}{l}\text { Yield } \\
(\%)\end{array}$ \\
\hline Boc-Ser-OMe, 1a & Boc- $\Delta \mathrm{Ala}(N$-Boc)-OMe, $\mathbf{2 a}$ & 92 \\
\hline Z-Ser-OMe, $\mathbf{1 b}$ & $\mathrm{Z}-\Delta \mathrm{Ala}(N$-Boc)-OMe, $\mathbf{2 b}$ & 85 \\
\hline $\mathrm{Z}\left(\mathrm{NO}_{2}\right)-$ Ser-OMe, $\mathbf{1 c}$ & $\mathrm{Z}\left(\mathrm{NO}_{2}\right)-\Delta \mathrm{Ala}(N-\mathrm{Boc})-\mathrm{OMe}, \mathbf{2 c}$ & 93 \\
\hline $\mathrm{Z}\left(\mathrm{NO}_{2}\right)-$ Ser-OBn, $\mathbf{1 d}$ & $\mathrm{Z}\left(\mathrm{NO}_{2}\right)-\Delta \mathrm{Ala}(N-\mathrm{Boc})-\mathrm{OBn}, \mathbf{2 d}$ & 91 \\
\hline Tos-Ser-OMe, $\mathbf{1 e}$ & Tos- $\Delta$ Ala $(N$-Boc $)-O M e, 2 \mathrm{e}$ & 99 \\
\hline Tos-Ser-OBn, $\mathbf{1 f}$ & Tos- $\Delta \mathrm{Ala}(N$-Boc)-OBn, $\mathbf{2 f}$ & 97 \\
\hline Bz-Ser-OMe, 1g & $\mathrm{Bz}-\Delta \mathrm{Ala}(N-\mathrm{Boc})-\mathrm{OMe}, \mathbf{2 g}$ & 92 \\
\hline Bz-Ser-OBn, $\mathbf{1 h}$ & $\mathrm{Bz}-\Delta \mathrm{Ala}(N-\mathrm{Boc})-\mathrm{OBn}, \mathbf{2 h}$ & 92 \\
\hline Boc-Thr-OMe, 1i & Boc- $\Delta \mathrm{ABu}(N$-Boc)-OMe, $2 \mathbf{i}$ & 87 \\
\hline Z-Thr-OMe, $\mathbf{1 j}$ & $\mathrm{Z}-\Delta \mathrm{Abu}(N$-Boc)-OMe, $\mathbf{2 j}$ & 94 \\
\hline $\mathrm{Z}\left(\mathrm{NO}_{2}\right)$-Thr-OMe, $\mathbf{1 k}$ & $\mathrm{Z}\left(\mathrm{NO}_{2}\right)-\Delta \mathrm{Abu}(N$-Boc)-OMe, $\mathbf{2 k}$ & 92 \\
\hline Tos-Thr-OMe, $\mathbf{1 l}$ & Tos- $\Delta \mathrm{Abu}(N$-Boc)-OMe, $2 \mathbf{l}$ & 87 \\
\hline $\begin{array}{l}\mathrm{Z}\left(\mathrm{NO}_{2}\right)-\mathrm{Phe}(\beta-\mathrm{OH})-\mathrm{OMe}, \\
\mathbf{1} \mathbf{m}\end{array}$ & $\mathrm{Z}\left(\mathrm{NO}_{2}\right)-\Delta \mathrm{Phe}(N$-Boc)-OMe, $\mathbf{2 m}$ & 93 \\
\hline Boc-Ser-OH, 1n & Boc- $\Delta \mathrm{Ala}(N-\mathrm{Boc})-\mathrm{OBu}^{t}, \mathbf{2 n}$ & 73 \\
\hline Boc-Thr-OH, 10 & Boc- $\Delta \mathrm{Abu}(N$-Boc $)-\mathrm{OBu}^{t}, \mathbf{2 o}$ & 73 \\
\hline H-Ser-OMe, 1p & Boc- $\Delta \mathrm{Ala}(N$-Boc)-OMe, $2 \mathbf{a}$ & 82 \\
\hline
\end{tabular}

$$
\begin{aligned}
\mathrm{R} & =\mathrm{H}, \mathrm{Me}, \mathrm{Ph} ; \\
\mathrm{R}^{1} & =\mathrm{H}, \mathrm{BOC}, \mathrm{Z}, \mathrm{Z}\left(\mathrm{NO}_{2}\right), \mathrm{TOS}, \mathrm{Bz} ; \\
\mathrm{R}^{2} & =\mathrm{H}, \mathrm{Me}, \mathrm{Bn}
\end{aligned}
$$

Scheme 1

bulkiness of the groups bound to the nitrogen atom, which would force and thus facilitate a trans $\mathrm{E}_{2}$-elimination. This is in agreement with results obtained by Srinivasan et $a .^{20}$ who have reported that base induced $\beta$-elimination of $N$-acyl-DL-Thr$\left(O\right.$-Tos)-OMe (threo type) proceeds via a trans $\mathrm{E}_{2}$-elimination to give the $Z$-isomer but only in a $70 \%$ yield. As shown in Table 1 , in all cases but one, the starting material had the amine function previously protected with one of the groups mentioned above, or by the benzoyl group, and except for two cases the carboxy function was protected as the methyl or the benzyl ester.

Recently, Nugent has patented a method for dehydration of $N$-acyl- $\beta$-hydroxyamino acid esters by treatment with an excess of acetic anhydride in the presence of pyridine. ${ }^{21}$ In this reaction an acetyl group was introduced at the amide function to give the $N$-acetyl- $N$-acyl-dehydroamino acid ester. The second acyl group bonded to the nitrogen atom would help formation of the new double bond, but the reported yields are only fair $(\approx 60 \%)$ and the product thus obtained is of limited value: the acetyl group cannot be easily removed and removal of both acyl groups would lead to decomposition of the hydroxyamino acid at the $\mathrm{N}-\mathrm{C}$ bond. ${ }^{2}$

The high yields obtained in the dehydration of serine derivatives by our method show the importance of increasing the bulk of the second acyl group at the nitrogen function. In fact, by sampling the reaction mixture throughout the preparation of compound $\mathbf{2 c}$, it was found that the reaction proceeds with formation of a tert-butyl carbonate, which undergoes $\beta$-elimination to the final product after a tert-butyloxycarbonyl group has been bound to the amine function. In an attempt to use an $N$-trityl serine derivative (Trt-Ser-OMe) as substrate for $\beta$-elimination the only product obtained was $\operatorname{Trt}-\operatorname{Ser}(O-\mathrm{Boc})-$ OMe. Steric hindrance related to the trityl group prevented further reaction at the nitrogen atom; the absence of dehydration suggests that, in addition to bulkiness at the nitrogen atom, the missing acyl group might be required as a driving force for elimination by stabilisation of the resulting $\alpha-\beta$ double bond.
Application of our methodology to an $N$-fluoren-9-ylmethoxycarbonyl (Fmoc) derivative of serine failed to give Fmoc$\Delta \mathrm{Ala}(N$-Boc)-OMe due to base induced cleavage of Fmoc by the catalyst (DMAP); when excess (Boc) $)_{2} \mathrm{O}$ was used, the Fmoc group was substituted by a further Boc group and, thus, the only product obtained was Boc- $\Delta \mathrm{Ala}(N$-Boc)-OMe.

DMAP catalysed esterifications with dicarbonate have been described by Takeda et $a l .{ }^{22}$ With the aim of simplifying our procedure by avoiding one of the two otherwise required protection steps, $N$-Boc protected serine and threonine having a free carboxy function were reacted with 3 eq. of tert-butyl pyrocarbonate in the presence of DMAP. As expected, both dehydration and esterification were obtained in the same step to give the tert-butyl ester of the $N, N$-(Boc) $)_{2}$-dehydroamino acid. However, the reactions were more sluggish and the yields slightly lower when compared to those of dehydration of the corresponding methyl or benzyl esters. Having again in mind to simplify our procedure by avoiding one of the two required protection steps, we have also investigated the direct dehydration of the methyl ester of serine. The reaction of this amino acid derivative with 3 eq. of $(\mathrm{Boc})_{2} \mathrm{O}$ allowed the preparation of 2a in an $82 \%$ yield, which was lower by $10 \%$ than that obtained when the $N$-Boc methyl ester of serine was used as starting material.

As stated previously, the use of most of the protecting groups was intended to allow the investigation of their cleavage from dehydroamino acids by mild electrolysis, ${ }^{16,17}$ which offers a clean, non-polluting alternative to the classical methods of reduction. Thus, both $\mathrm{Z}\left(\mathrm{NO}_{2}\right)$ and Tos were selectively removed by electrolysis at controlled potential from $\mathbf{2 c}$ and $\mathbf{2 e}$ to give Boc- $\triangle \mathrm{Ala}-\mathrm{OMe}$ in yields of 88 and $73 \%$, respectively, and from $\mathbf{2 k}$ and $2 \mathbf{2}$ to give Boc- $\triangle \mathrm{Abu}-\mathrm{OMe}$ in yields of 88 and $78 \%$, respectively. However, when electrochemical equipment is not available selective cleavage can still be achieved by taking advantage of an alternative strategy we have developed recently, ${ }^{23}$ which is based on selective reduction with an appropriate metal according to the potential required. As an example, $\mathrm{Z}\left(\mathrm{NO}_{2}\right)$ was cleaved by selective reduction from $\mathbf{2 c}$ and $\mathbf{2 k}$ with mercury activated aluminium to give Boc- $\Delta \mathrm{Ala}-\mathrm{OMe}$ and Boc- $\triangle \mathrm{Abu}-\mathrm{OMe}$ in yields of 87 and $95 \%$, respectively.

Cleavage of Boc from the diacyl derivatives was also easily performed with trifluoroacetic acid (TFA) and, thus, $\mathbf{2} \mathbf{j}$ and $\mathbf{2 k}$ gave $\mathrm{Z}-\Delta \mathrm{Abu}-\mathrm{OMe}$ and $\mathrm{Z}\left(\mathrm{NO}_{2}\right)-\Delta \mathrm{Abu}-\mathrm{OMe}$ in yields of 87 and $85 \%$, respectively. Z- $\Delta$ Abu-OMe was saponified to yield $77 \%$ of $\mathrm{Z}-\Delta \mathrm{Abu}-\mathrm{OH}$, which was then coupled with glycine methyl ester by dicyclohexylcarbodiimide-hydroxybenzotriazole (DCC$\mathrm{HOBt}$ ) coupling to give the corresponding dehydrodipeptide in an $85 \%$ yield.

Owing to the low reactivity of the $\alpha$-amine group of dehydroalanine ${ }^{24}$ and to the instability of its $\mathrm{N}$-deprotected derivatives, ${ }^{2}$ most of the dehydroamino acid derivatives mentioned so far are of limited application in peptide synthesis. This led us to investigate the applicability of our methodology to the dehydration of peptides containing $\beta$-hydroxyamino acids (3a-g, Table 2) as precursors of dehydropeptides (4a-g). Thus, dipeptides containing serine or threonine in either the amine or the carboxy terminus were reacted under the conditions described previously. In these reactions 3 eq. of (Boc) ${ }_{2} \mathrm{O}$ were used, i.e. 2 eq. for acylation of both amide nitrogen atoms and a third equivalent to generate the carbonate at the $\beta$-carbon atom, which subsequently underwent elimination to form the double bond. Again, peptides containing threonine gave only one of the two possible geometric isomers. In the case of a dipeptide containing both threonine and serine (3e) and of another containing two residues of threonine (3f), simultaneous dehydration of both amino acid residues was achieved.

In conclusion, the method reported above allows the high yield preparation of a variety of dehydroamino acid and dehydroamino acid-containing peptide derivatives by $\beta$-elimination of the respective $\beta$-hydroxyamino acid or $\beta$-hydroxy- 
Table 2 Results obtained in the synthesis of dehydropeptide derivatives

\begin{tabular}{|c|c|c|}
\hline Reagent & Product & $\begin{array}{l}\text { Yield } \\
(\%)\end{array}$ \\
\hline $\begin{array}{l}\mathrm{Z}\left(\mathrm{NO}_{2}\right)-\text { Ser-Phe-OEt, } \\
\text { 3a }\end{array}$ & $\begin{array}{l}\mathrm{Z}\left(\mathrm{NO}_{2}\right)-\Delta \mathrm{Ala}(N-\mathrm{Boc})-\mathrm{Phe}(N-\mathrm{Boc})- \\
\mathrm{OEt}, \mathbf{4 a}\end{array}$ & 93 \\
\hline Boc-Ala-Ser-OMe, 3b & $\begin{array}{l}\operatorname{Boc}-\mathrm{Ala}(N-\mathrm{Boc})-\Delta \mathrm{Ala}(N-\mathrm{Boc})-\mathrm{OMe}, \\
\text { 4b }\end{array}$ & 91 \\
\hline Z-Thr-Gly-OMe, 3c & $\mathrm{Z}$-Abu( $N$-Boc)-Gly( $N$-Boc)-OMe, $\mathbf{4 c}$ & 81 \\
\hline Boc-Ala-Thr-OMe, 3d & $\begin{array}{l}\text { Boc-Ala }(N-\mathrm{Boc})-\Delta \mathrm{Abu}(N-\mathrm{Boc})-\mathrm{OMe}, \\
\text { 4d }\end{array}$ & 84 \\
\hline Boc-Thr-Ser-OMe, $\mathbf{3 e}$ & $\begin{array}{l}\text { Boc- } \Delta \mathrm{Abu}(N-\mathrm{Boc})-\Delta \mathrm{Ala}(N-\mathrm{Boc})- \\
\mathrm{OMe}, \mathbf{4 e}\end{array}$ & 83 \\
\hline $\begin{array}{l}\text { Boc-Thr-Thr-OMe, } \\
\text { 3f }\end{array}$ & $\begin{array}{l}\text { Boc- } \Delta \mathrm{Abu}(N \text {-Boc })-\Delta \mathrm{Abu}(N-\mathrm{Boc})- \\
\mathrm{OMe}, \mathbf{4 f}\end{array}$ & 74 \\
\hline
\end{tabular}

amino acid containing peptides using mild reaction conditions and simple work-up procedures. In the case of dehydration of $\beta$-alkyl or $\beta$-aryl substituted $\beta$-hydroxyamino acids, unlike other methods previously reported, this methodology offers the further advantage of leading to a single isomer.

\section{Experimental}

\section{General methods}

All melting points were determined on a Gallenkamp melting point apparatus and are uncorrected. TLC analyses were carried out on $0.25 \mathrm{~mm}$ thick precoated silica plates (Merck Fertigplatten Kieselgel $60 \mathrm{~F}_{254}$ ) and spots were visualised under UV light or, preferably, by heating and subsequent dicarboxidine-spray. Preparative chromatography was carried out on Merck Kieselgel 60 (230-400 mesh). Petroleum ether refers to the light petroleum fraction of boiling range $40-60{ }^{\circ} \mathrm{C} .{ }^{1} \mathrm{H}$ NMR spectra were recorded on a Varian $300 \mathrm{MHz}$ spectrometer for $\sim 5 \% \mathrm{CDCl}_{3}$ solutions at $25{ }^{\circ} \mathrm{C}$, unless otherwise stated. All shifts are given in $\delta$ ppm using $\delta_{\mathrm{H}}\left(\mathrm{Me}_{4} \mathrm{Si}\right)=0$ as reference. $J$ values are given in $\mathrm{Hz}$. Assignments were made by comparison of chemical shifts, peak multiplicities and $J$ values. ${ }^{13} \mathrm{C}$ NMR spectra were determined for $\mathrm{CDCl}_{3}$ solutions in the same instrument at $75.4 \mathrm{MHz}$ using the solvent peak as internal reference. Elemental analyses of crystalline derivatives and some oils were carried out on a Leco CHNS 932 instrument. For controlled potential electrolysis experiments a Hi-Tek potentiostat DT 2101, and a Hi-Tek wave generator PP RI, connected to a Philips recorder PM 8043 were used. The electrolysis cell was a conventional two-compartment, threeelectrode, home-built batch cell of the type illustrated elsewhere. $^{16}$

\section{Preparation of $\mathrm{N}$-acyl amino acid esters}

Synthesis of 1a. $\mathrm{H}-\mathrm{Ser}-\mathrm{OMe} \cdot \mathrm{HCl}$ was dissolved in dichloromethane $\left(1 \mathrm{~mol} \mathrm{dm}^{-3}\right)$ and 2.2 eq. of triethylamine added, then 1.1 eq. of tert-butylpyrocarbonate were slowly added with vigorous stirring and cooling in an ice bath. After stirring at $0{ }^{\circ} \mathrm{C}$ for $30 \mathrm{~min}$ the solution was stirred at room temperature for $3 \mathrm{~h}$. The reaction mixture was then evaporated and partitioned between $200 \mathrm{~cm}^{3}$ of ethyl acetate and $100 \mathrm{~cm}^{3}$ of $\mathrm{KHSO}_{4}(1 \mathrm{~mol}$ $\left.\mathrm{dm}^{-3}\right)$ and washed with $\mathrm{KHSO}_{4}\left(1 \mathrm{~mol} \mathrm{dm}^{-3}\right), \mathrm{NaHCO}_{3}(1 \mathrm{~mol}$ $\mathrm{dm}^{-3}$ ) and brine (3 times, $50 \mathrm{~cm}^{3}$ each). After drying over $\mathrm{MgSO}_{4}$ the extract was taken to dryness at reduced pressure to give 1a $(85 \%)$, oil (lit., ${ }^{25}$ oil); $\delta_{\mathrm{H}} 1.46\left(9 \mathrm{H}, \mathrm{s}, \mathrm{CH}_{3}\right.$ Boc), 3.79 (3 $\left.\mathrm{H}, \mathrm{s}, \mathrm{CH}_{3} \mathrm{OMe}\right), 3.88-4.98$ ( $2 \mathrm{H}$, complex, $\left.\mathrm{\beta CH}_{2} \mathrm{Ser}\right), 4.39$ $(1 \mathrm{H}, \mathrm{m}, \alpha \mathrm{C} H \mathrm{Ser}), 5.47(1 \mathrm{H}, \mathrm{br}, \alpha \mathrm{N} H) ; \delta_{\mathrm{C}} 28.24,52.57,55.69$, $63.38,80.30,155.72,171.34$.

Synthesis of 1b, 1e and 1g. The same procedure as described for the preparation of 1a was used substituting benzyl chloroformate, tosyl chloride and benzoyl chloride respectively for tert-butyl pyrocarbonate to afford the $N$-acyl serine methyl esters: 1b, mp $42.5-44.1^{\circ} \mathrm{C}$ (from ethyl acetate- $n$-hexane) (lit., ${ }^{26}$ $33-35^{\circ} \mathrm{C}$ ); 1e, mp $87-88^{\circ} \mathrm{C}$ (from ethyl acetate-petroleum ether) (lit., ${ }^{27} 92-93{ }^{\circ} \mathrm{C}$ ); $\mathbf{1 g}, \mathrm{mp} 84.5-85.5^{\circ} \mathrm{C}$ (from ethyl acetate-petroleum ether) (lit., ${ }^{28} 84-86^{\circ} \mathrm{C}$ ).

Synthesis of 1c. The same procedure as described for the preparation of 1a was followed substituting $p$-nitrobenzyl chloroformate for tert-butyl pyrocarbonate to give $1 \mathbf{c}^{29}(71 \%)$, $\mathrm{mp} 74-75^{\circ} \mathrm{C}$ (from ethyl acetate-petroleum ether) (Found: $\mathrm{C}$, 48.1; $\mathrm{H}, 4.7 ; \mathrm{N}, 9.6$. Calc. for $\mathrm{C}_{12} \mathrm{H}_{14} \mathrm{~N}_{2} \mathrm{O}_{7}$ : C, 48.3; H, 4.7; N, 9.4\%); $\delta_{\mathrm{H}} 3.81\left(3 \mathrm{H}, \mathrm{s}, \mathrm{CH}_{3} \mathrm{OMe}\right), 3.91-4.09(2 \mathrm{H}$, complex, $\left.\mathrm{\beta CH}_{2} \mathrm{Ser}\right), 4.46(1 \mathrm{H}, \mathrm{m}, \alpha \mathrm{CH} \mathrm{Ser}), 5.24\left[2 \mathrm{H}, \mathrm{s}, \mathrm{CH}_{2} \mathrm{Z}\left(\mathrm{NO}_{2}\right)\right]$, $5.80(1 \mathrm{H}, \mathrm{d}, J 7.0, \alpha \mathrm{NH}), 7.53,8.23[2 \mathrm{H}+2 \mathrm{H}, 2 \mathrm{~d}, J 9.0, \operatorname{Ar} H$ $\left.\mathrm{Z}\left(\mathrm{NO}_{2}\right)\right] ; \delta_{\mathrm{C}} 52.86,56.00,63.15,65.61,123.78,128.12,134.02$, $143.48,147.63,156.26,170.74$.

Preparation of 1d. The same procedure as described for the preparation of 1c was followed substituting $\mathrm{H}-\mathrm{Ser}-\mathrm{OBn} \cdot \mathrm{HCl}$ for $\mathrm{H}-\mathrm{Ser}-\mathrm{OMe} \cdot \mathrm{HCl}$ to give $\mathbf{1 d}(76 \%), \mathrm{mp} 85-87^{\circ} \mathrm{C}$ (from ethyl acetate-petroleum ether) (Found: $\mathrm{C}, 57.6 ; \mathrm{H}, 4.6 ; \mathrm{N}, 7.2$. Calc. for $\left.\mathrm{C}_{18} \mathrm{H}_{18} \mathrm{~N}_{2} \mathrm{O}_{7}: \mathrm{C}, 57.8 ; \mathrm{H}, 4.8 ; \mathrm{N}, 7.5 \%\right) ; \delta_{\mathrm{H}} 4.01(2 \mathrm{H}, \mathrm{m}$, $\left.\mathrm{\beta CH}_{2} \mathrm{Ser}\right), 4.50\left(1 \mathrm{H}, \mathrm{m}, \alpha \mathrm{CH}_{2} \mathrm{Ser}\right), 5.22,5.24[2 \mathrm{H}+2 \mathrm{H}, 2 \mathrm{~s}$, $\mathrm{CH}_{2} \mathrm{Z}\left(\mathrm{NO}_{2}\right)$ and $\mathrm{CH}_{2} \mathrm{Bn}$ ], $5.78(1 \mathrm{H}, \mathrm{br}, \alpha \mathrm{NH}), 7.37(5 \mathrm{H}, \mathrm{s}$, $\mathrm{Ar} H \mathrm{Bn}), 7.51,8.21\left[2 \mathrm{H}+2 \mathrm{H}, 2 \mathrm{~d}, J\right.$ 8.4, $\left.\mathrm{ArH} \mathrm{Z}\left(\mathrm{NO}_{2}\right)\right]$; $\delta_{\mathrm{C}}\left(75.4 \mathrm{MHz} ; \mathrm{CDCl}_{3}\right) 56.15,63.14,65.56,67.62,123.75,128.06$, $128.18,128.61,134.98,143.48,147.63,155.70,170.18$.

Preparation of 1f. The same procedure as described for the preparation of 1d was followed substituting tosyl chloride for p-nitrobenzyl chloroformate to give $\mathbf{1 f}^{15}(87 \%), \mathrm{mp} 82-83^{\circ} \mathrm{C}$ (from ethyl acetate-petroleum ether) (Found: C, 58.6; H, 5.4; $\mathrm{N}, 4.1 ; \mathrm{S}, 9.2$. Calc. for $\mathrm{C}_{17} \mathrm{H}_{19} \mathrm{NO}_{5} \mathrm{~S}: \mathrm{C}, 58.4 ; \mathrm{H}, 5.5 ; \mathrm{N}, 4.0 ; \mathrm{S}$, $9.2 \%) ; \delta_{\mathrm{H}} 2.41\left(3 \mathrm{H}, \mathrm{s}, \mathrm{CH}_{3} \mathrm{Tos}\right), 3.91\left(2 \mathrm{H}, \mathrm{m}, \mathrm{\beta CH}_{2} \mathrm{Ser}\right), 4.04$ $(1 \mathrm{H}, \mathrm{m}, \alpha \mathrm{CH}$ Ser $), 5.04\left(2 \mathrm{H}, \mathrm{s}, \mathrm{CH}_{2} \mathrm{Bn}\right), 7.27(5 \mathrm{H}, \mathrm{m}, \mathrm{Ar} H$ $\mathrm{Bn}), 7.35,7.73(2 \mathrm{H}+2 \mathrm{H}, 2 \mathrm{~d}, J 8.1, \mathrm{Ar} H \mathrm{Tos}) ; \delta_{\mathrm{C}} 21.55,57.68$, $63.78,65.36,127.16,128.12,128.38,128.54,129.75,134.67$, $136.38,143.84,169.53$.

Preparation of $\mathbf{1 h}$. The same procedure as described for the preparation of 1d was followed substituting benzoyl chloride for $p$-nitrobenzyl chloroformate to give $\mathbf{1 h}^{30}(97 \%)$, mp 105$106^{\circ} \mathrm{C}$ (from ethyl acetate- $n$-hexane) (Found: C, 68.5; H, 5.8; $\mathrm{N}$, 4.6. Calc. for $\mathrm{C}_{17} \mathrm{H}_{17} \mathrm{NO}_{4}$ : C, 68.2; $\left.\mathrm{H}, 5.7 ; \mathrm{N}, 4.7 \%\right) ; \delta_{\mathrm{H}} 4.02-$ 4.12 (2 H, complex, $\left.\beta \mathrm{CH}_{2} \mathrm{Ser}\right), 4.91\left(1 \mathrm{H}, \mathrm{m}, \alpha \mathrm{CH}_{2} \mathrm{Ser}\right), 5.25$ $\left(2 \mathrm{H}, \mathrm{s}, \mathrm{CH}_{2} \mathrm{Bn}\right), 7.18(1 \mathrm{H}, \mathrm{d}, J 6.9, \alpha \mathrm{NH}), 7.35-7.84(10 \mathrm{H}$, complex, $\operatorname{Ar} H \mathrm{Bz}+\operatorname{Ar} H \mathrm{Bn}) ; \delta_{\mathrm{C}} 55.29,63.47,67.61,127.15$, $128.15,128.53,128.64,131.94,133.46,135.07,167.70,170.44$.

Preparation of 1i. The same procedure as described for preparation of 1a was followed substituting $\mathrm{H}-\mathrm{Thr}-\mathrm{OMe} \cdot \mathrm{HCl}$ for $\mathrm{H}$-Ser-OMe $\cdot \mathrm{HCl}$ to give $\mathbf{1 i}{ }^{31}(76 \%)$, oil, $\delta_{\mathrm{H}} 1.25(3 \mathrm{H}, \mathrm{d}, J 6.3$, $\left.\gamma \mathrm{CH}_{3} \mathrm{Thr}\right), 1.46\left(9 \mathrm{H}, \mathrm{s}, \mathrm{CH}_{3} \mathrm{Boc}\right), 3.78\left(3 \mathrm{H}, \mathrm{s}, \mathrm{CH}_{3} \mathrm{OMe}\right), 4.28$ $(2 \mathrm{H}, \mathrm{m}, \alpha \mathrm{CH} \mathrm{Thr}$ and $\beta \mathrm{CH} \mathrm{Thr}), 5.33(1 \mathrm{H}, \mathrm{br}, \alpha \mathrm{NH})$; $\delta_{\mathrm{C}} 19.84,28.25,52.46,58.66,60.08,80.30,156.20,171.97$.

Preparation of $\mathbf{1 j}$. The same procedure as described for preparation of 1i was followed substituting benzyl chloroformate for tert-butyl pyrocarbonate to give $\mathbf{1 j}^{20}$ (74\%), mp $90-91{ }^{\circ} \mathrm{C}$ (from ethyl acetate-diethyl ether) (Found: C, 58.3; H, 6.6; N, 5.3. Calc. for $\mathrm{C}_{13} \mathrm{H}_{17} \mathrm{NO}_{5}$ : C, 58.4; H, 6.4; N, 5.2\%); $\delta_{\mathrm{H}} 1.26$ (3 H, d, $J$ 6.3, $\left.\gamma \mathrm{CH} H_{3} \mathrm{Thr}\right), 3.78\left(3 \mathrm{H}, \mathrm{s}, \mathrm{CH}_{3} \mathrm{OMe}\right), 4.34(2 \mathrm{H}$, $\mathrm{m}, \alpha \mathrm{CH}$ Thr and $\beta \mathrm{CH} \mathrm{Thr}), 5.14\left(2 \mathrm{H}, \mathrm{s}, \mathrm{CH}_{2} \mathrm{Z}\right), 5.57(1 \mathrm{H}, \mathrm{d}$, $J$ 8.1, $\alpha \mathrm{N} H), 7.35(5 \mathrm{H}, \mathrm{m}, \operatorname{Ar} H \mathrm{Z}) ; \delta_{\mathrm{C}} 16.78,49.49,56.06$, 64.13, 64.88, 124.95, 125.11, 125.44, 133.08, 153.63, 168.56.

Preparation of $1 \mathbf{k}$. The same procedure as described for preparation of $\mathbf{1 i}$ was followed substituting $p$-nitrobenzyl chloroformate for tert-butyl pyrocarbonate to give $\mathbf{1 k}(97 \%)$, $\mathrm{mp} 63.5-65^{\circ} \mathrm{C}$ (from diethyl ether- $n$-hexane) (Found: C, 50.0; 
$\mathrm{H}, 5.1 ; \mathrm{N}$, 8.9. Calc. for $\mathrm{C}_{13} \mathrm{H}_{16} \mathrm{~N}_{2} \mathrm{O}_{7}$ : C, 50.0; $\mathrm{H}, 5.2 ; \mathrm{N}, 9.0 \%$ ); $\delta_{\mathrm{H}} 1.27\left(3 \mathrm{H}, \mathrm{d}, J 6.6, \gamma \mathrm{CH}_{3} \mathrm{Thr}\right), 3.79\left(3 \mathrm{H}, \mathrm{s}, \mathrm{CH}_{3} \mathrm{OMe}\right), 4.30$ $4.38(2 \mathrm{H}$, complex, $\alpha \mathrm{CH}$ Thr and $\beta \mathrm{CH} \mathrm{Thr}), 5.24\left[2 \mathrm{H}, \mathrm{s}, \mathrm{CH}_{2}\right.$ $\left.\mathrm{Z}\left(\mathrm{NO}_{2}\right)\right], 5.69(1 \mathrm{H}, \mathrm{d}, J 8.4, \alpha \mathrm{NH}), 7.53,8.22[2 \mathrm{H}+2 \mathrm{H}, 2 \mathrm{~d}$, $J$ 8.7, $\left.\mathrm{Ar} H \mathrm{Z}\left(\mathrm{NO}_{2}\right)\right] ; \delta_{\mathrm{C}} 19.92,52.65,59.16,65.56,67.77$, $123.71,127.96,143.63,147.56,156.23,171.46$.

Preparation of 11. The same procedure as described for preparation of $\mathbf{1 i}$ was followed substituting tosyl chloride for tertbutyl pyrocarbonate to give $1 \mathrm{1I}^{32}$ (92\%), mp 99-100 ${ }^{\circ} \mathrm{C}$ (from ethyl acetate-petroleum ether) (Found: C, 50.2; H, 6.1; N, 4.9; $\mathrm{S}$, 11.3. Calc. for $\mathrm{C}_{12} \mathrm{H}_{17} \mathrm{NO}_{5} \mathrm{~S}: \mathrm{C}, 50.2 ; \mathrm{H}, 6.0 ; \mathrm{N}, 4.9 ; \mathrm{S}, 11.1 \%$ ); $\delta_{\mathrm{H}} 1.27\left(3 \mathrm{H}, \mathrm{d}, J 6.6, \gamma \mathrm{CH}_{3} \mathrm{Thr}\right), 2.42\left(3 \mathrm{H}, \mathrm{s}, \mathrm{CH}_{3} \mathrm{Tos}\right), 3.53$ ( $3 \mathrm{H}, \mathrm{s}, \mathrm{CH}_{3} \mathrm{OMe}$ ), 3.83 (2 H, dd, $J$ 3.0, $J$ 9.3, $\beta \mathrm{CH} \mathrm{Thr}$ ), 4.15 $(1 \mathrm{H}, \mathrm{m}, \alpha \mathrm{CH} \mathrm{Thr}), 5.53(1 \mathrm{H}, \mathrm{br}, \alpha \mathrm{NH}), 7.30,7.72(2 \mathrm{H}+2 \mathrm{H}$, d, $J$ 8.1, Ar $H$ Tos); $\delta_{\mathrm{C}} 19.79,21.51,52.59,60.93,68.36,127.21$, 129.58, 136.69, 143.69, 170.69 .

Preparation of $1 \mathbf{m}$. The same procedure as described for preparation of 1c was followed substituting $\mathrm{H}-\mathrm{Ph}(\beta-\mathrm{OH})$ $\mathrm{OMe} \cdot \mathrm{HCl}$ for $\mathrm{H}-\mathrm{Ser}-\mathrm{OMe} \cdot \mathrm{HCl}$ to give $\mathbf{1 m}(82 \%)$, mp 114 $115{ }^{\circ} \mathrm{C}$ (from ethyl acetate-petroleum ether) (Found: C, 57.95; $\mathrm{H}, 4.3 ; \mathrm{N}, 7.5$. Calc. for $\mathrm{C}_{18} \mathrm{H}_{18} \mathrm{~N}_{2} \mathrm{O}_{7}$ : C, 58.1; $\mathrm{H}, 4.3 ; \mathrm{N}, 7.5 \%$ ); $\delta_{\mathrm{H}} 3.80\left(3 \mathrm{H}, \mathrm{s}, \mathrm{CH}_{3} \mathrm{OMe}\right), 4.62\left[1 \mathrm{H}, \mathrm{dd}, J 2.4, J 9.5, \beta \mathrm{CH}_{2}\right.$ Phe $(\beta-\mathrm{OH})], 5.10\left[2 \mathrm{H}, \mathrm{q}, J 6.9, \mathrm{CH}_{2} \mathrm{Z}\left(\mathrm{NO}_{2}\right)\right], 5.34[1 \mathrm{H}, \mathrm{q}$, $J$ 2.4, $\alpha \mathrm{CH}$ Phe $(\beta-\mathrm{OH})], 5.71(1 \mathrm{H}, \mathrm{d}, J 6.6, \alpha \mathrm{NH}), 7.35$ $\left[5 \mathrm{H}+2 \mathrm{H}, \mathrm{m}, \operatorname{Ar} H \mathrm{Phe}(\beta-\mathrm{OH})+\operatorname{Ar} H \mathrm{Z}\left(\mathrm{NO}_{2}\right)\right], 8.67[2 \mathrm{H}$, d, $J$ 8.7, $\left.\operatorname{Ar} H \mathrm{Z}\left(\mathrm{NO}_{2}\right)\right] ; \delta_{\mathrm{C}} 52.77,59.79,65.33,73.38,123.63$, $125.75,126.95,127.77,128.18,128.45,139.45,143.69,155.73$, 170.90 .

\section{Synthesis of $N$-Boc, $N$-acyl dehydroamino acid esters}

Synthesis of 2a. To a solution of Boc-Ser-OMe in dry acetonitrile $\left(1 \mathrm{~mol} \mathrm{dm}^{-3}\right), 0.1$ eq. of DMAP was added followed by 2.2 eq. of tert-butyl pyrocarbonate under rapid stirring at room temperature. The reaction was stirred for $12 \mathrm{~h}$ while monitored by TLC (diethyl ether- $n$-hexane, $1: 1$ ). Evaporation under reduced pressure gave a residue that was partitioned between $200 \mathrm{~cm}^{3}$ of diethyl ether and $100 \mathrm{~cm}^{3}$ of $\mathrm{KHSO}_{4}(1 \mathrm{~mol}$ $\mathrm{dm}^{-3}$ ). The organic phase was thoroughly washed with $\mathrm{KHSO}_{4}$

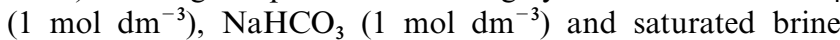
$\left(3 \times 50 \mathrm{~cm}^{3}\right.$ each), and dried over $\mathrm{MgSO}_{4}$. Removal of the solvent afforded pure $\mathbf{2} \mathbf{a}^{8}(92 \%)$.

Synthesis of $\mathbf{2 b}$. The same procedure as described for the preparation of 2a was followed substituting Z-Ser-OMe for Boc-Ser-OMe to give $\mathbf{2 b}$. Since attempts to crystallise the compound were not successful the dehydroamino acid derivative was chromatographed on silica using diethyl ether- $n$-hexane as eluent to give $\mathbf{2 b}$ as a pure oil $(85 \%$ ) (Found: $\mathrm{C}, 60.8 ; \mathrm{H}, 6.4 ; \mathrm{N}$, 4.2. Calc. for $\mathrm{C}_{17} \mathrm{H}_{21} \mathrm{NO}_{6}$ : C, 60.9; $\left.\mathrm{H}, 6.3 ; \mathrm{N}, 4.2 \%\right) ; \delta_{\mathrm{H}} 1.46(9$ $\left.\mathrm{H}, \mathrm{s}, \mathrm{CH}_{3} \mathrm{Boc}\right), 3.73\left(3 \mathrm{H}, \mathrm{s}, \mathrm{CH}_{3} \mathrm{OMe}\right), 5.22\left(2 \mathrm{H}, \mathrm{s}, \mathrm{CH}_{2}\right.$ $\mathrm{Z}), 5.70,6.41\left(1 \mathrm{H}+1 \mathrm{H}, 2 \mathrm{~s}, \beta \mathrm{CH}_{2}\right), 7.34(5 \mathrm{H}, \mathrm{m}, \operatorname{Ar} H \mathrm{Z})$; $\delta_{\mathrm{C}} 27.76,52.40,68.41,83.72,125.59,127.98,128.27,128.46$, $135.19,135.50,150.13,151.96,163.64$.

Synthesis of 2c. The same procedure as described for the preparation of $\mathbf{2 a}$ was followed substituting $\mathrm{Z}\left(\mathrm{NO}_{2}\right)$-Ser-OMe for Boc-Ser-OMe to give $2 \mathrm{c}(93 \%)$, mp $97-98^{\circ} \mathrm{C}$ (from diethyl ether- $n$-hexane) (Found: $\mathrm{C}, 53.7 ; \mathrm{H}, 5.3 ; \mathrm{N}, 7.0$. Calc. for $\left.\mathrm{C}_{17} \mathrm{H}_{20} \mathrm{~N}_{2} \mathrm{O}_{8}: \mathrm{C}, 53.7 ; \mathrm{H}, 5.3 ; \mathrm{N}, 7.4 \%\right) ; \delta_{\mathrm{H}} 1.47\left(9 \mathrm{H}, \mathrm{s}, \mathrm{CH}_{3}\right.$ Boc), 3.79 (3 H, s, $\left.\mathrm{CH}_{3} \mathrm{OMe}\right), 5.32\left[2 \mathrm{H}, \mathrm{s}, \mathrm{CH}_{2} \mathrm{Z}\left(\mathrm{NO}_{2}\right)\right.$ ], 5.77, $6.46\left(1 \mathrm{H}+1 \mathrm{H}, 2 \mathrm{~s}, \beta \mathrm{CH}_{2}\right), 7.52,8.24[2 \mathrm{H}+2 \mathrm{H}, 2 \mathrm{~d}, J 9.0$, $\left.\mathrm{Ar} H \mathrm{Z}\left(\mathrm{NO}_{2}\right)\right] ; \delta_{\mathrm{C}} 27.74,52.56,66.86,84.12,123.74,125.89$, $128.05,135.39,142.47,147.74,149.78,151.83,163.50$.

Synthesis of 2d. The same procedure as described for the preparation of 2a was followed substituting $\mathrm{Z}\left(\mathrm{NO}_{2}\right)$-Ser-OBn for Boc-Ser-OMe to give 2d (81\%), mp 93-94 ${ }^{\circ} \mathrm{C}$ (from diethyl ether- $n$-hexane) (Found: C, 60.4; H, 5.3; N, 6.1. Calc. for $\left.\mathrm{C}_{23} \mathrm{H}_{24} \mathrm{~N}_{2} \mathrm{O}_{8}: \mathrm{C}, 60.5 ; \mathrm{H}, 5.3 ; \mathrm{N}, 6.1 \%\right) ; \delta_{\mathrm{H}} 1.42\left(9 \mathrm{H}, \mathrm{s}, \mathrm{CH}_{3}\right.$ Boc), 5.23, $5.26\left[2 \mathrm{H}+2 \mathrm{H}, 2 \mathrm{~s}, \mathrm{CH}_{2} \mathrm{OBn}+\mathrm{CH}_{2} \mathrm{Z}\left(\mathrm{NO}_{2}\right)\right]$, 5.79, $6.50\left(1 \mathrm{H}+1 \mathrm{H}, 2 \mathrm{~s}, \beta \mathrm{CH}_{2}\right), 7.34(5 \mathrm{H}, \mathrm{m}, \mathrm{Ar} H \mathrm{Bn}), 7.44$, $8.16\left[2 \mathrm{H}+2 \mathrm{H}, 2 \mathrm{~d}, J 8.4, \operatorname{Ar} H \mathrm{Z}\left(\mathrm{NO}_{2}\right)\right] ; \delta_{\mathrm{C}} 27.69,66.80,67.30$, $84.17,123.73,126.23,127.95,128.18,128.48,128.58,135.21$, 135.47, 142.40, 147.66, 149.78, 151.74, 162.88 .

Synthesis of 2e. The same procedure as described for the preparation of 2a was followed substituting Tos-Ser-OMe for Boc-Ser-OMe to give $\mathbf{2} \mathbf{e}^{19}(99 \%)$.

Synthesis of $2 \mathrm{f}$. The same procedure as described for the preparation of 2a was followed substituting Tos-Ser-OBn for Boc-Ser-OMe to give $2 \mathrm{f}(97 \%)$, mp $113-114^{\circ} \mathrm{C}$ (from diethyl ether- $n$-hexane) (Found: C, 61.4; H, 6.1; N, 3.45; S, 7.4. Calc. for $\left.\mathrm{C}_{22} \mathrm{H}_{25} \mathrm{NO}_{6} \mathrm{~S}: \mathrm{C}, 61.2 ; \mathrm{H}, 5.8 ; \mathrm{N}, 3.25 ; \mathrm{S}, 7.4 \%\right) ; \delta_{\mathrm{H}} 1.28(9 \mathrm{H}$, s, $\left.\mathrm{CH}_{3} \mathrm{Boc}\right), 2.42\left(3 \mathrm{H}, \mathrm{s}, \mathrm{CH}_{3} \mathrm{Tos}\right), 5.22\left(2 \mathrm{H}, \mathrm{s}, \mathrm{CH}_{2} \mathrm{OBn}\right)$, $6.09,6.67\left(1 \mathrm{H}+1 \mathrm{H}, 2 \mathrm{~s}, \beta \mathrm{CH} H_{2}\right), 7.23(2 \mathrm{H}, \mathrm{d}, J 8.1, \operatorname{Ar} H \mathrm{Tos})$, $7.34(5 \mathrm{H}, \mathrm{m}, \mathrm{Ar} H \mathrm{Bn}), 7.91(2 \mathrm{H}, \mathrm{d}, J 8.1, \mathrm{Ar} H \mathrm{Tos}) ; \delta_{\mathrm{C}} 21.62$, $27.70,67.46,84.82,128.21,128.35,128.52,129.02,129.04$, $130.02,133.25,135.13,136.04,144.49,149.64,163.03$.

Synthesis of $2 \mathrm{~g}$. The same procedure as described for the preparation of $\mathbf{2 a}$ was followed substituting Bz-Ser-OMe for Boc-Ser-OMe to give $2 \mathrm{~g}(92 \%), \mathrm{mp} 73-73.5^{\circ} \mathrm{C}$ (from diethyl ether- $n$-hexane) (Found: C, 63.0; H, 6.35; N, 4.6. Calc. for $\left.\mathrm{C}_{16} \mathrm{H}_{19} \mathrm{NO}_{5}: \mathrm{C}, 62.9 ; \mathrm{H}, 6.3 ; \mathrm{N}, 4.6 \%\right) ; \delta_{\mathrm{H}} 1.24\left(9 \mathrm{H}, \mathrm{s}, \mathrm{CH}_{3} \mathrm{Boc}\right)$, $3.84\left(3 \mathrm{H}, \mathrm{s}, \mathrm{CH}_{3} \mathrm{OMe}\right), 5.84,6.53\left(1 \mathrm{H}+1 \mathrm{H}, 2 \mathrm{~s}, \beta \mathrm{CH}_{2}\right), 7.42-$ $7.74(5 \mathrm{H}$, complex, $\mathrm{Ar} H \mathrm{Bz}) ; \delta_{\mathrm{C}} 27.38,52.60,83.83,125.90$, $128.14,131.73,136.00,136.25,151.96,163.68,172.18$.

Synthesis of $\mathbf{2 h}$. The same procedure as described for the preparation of 2a was followed substituting Bz-Ser-OBn for Boc-Ser-OMe to give $\mathbf{2 h}(92 \%)$, mp $76-77^{\circ} \mathrm{C}$ (from diethyl ether- $n$-hexane) (Found: C, 69.3; H, 6.2; N, 3.7. Calc. for $\left.\mathrm{C}_{22} \mathrm{H}_{23} \mathrm{NO}_{5}: \mathrm{C}, 69.3 ; \mathrm{H}, 6.1 ; \mathrm{N}, 3.7 \%\right) ; \delta_{\mathrm{H}} 1.19\left(9 \mathrm{H}, \mathrm{s}, \mathrm{CH}_{3} \mathrm{Boc}\right)$, $5.26\left(2 \mathrm{H}, \mathrm{s}, \mathrm{CH}_{2} \mathrm{OBn}\right), 5.87,6.58\left(1 \mathrm{H}+1 \mathrm{H}, 2 \mathrm{~s}, \mathrm{\beta CH}_{2}\right), 7.35-$ $7.68(10 \mathrm{H}$, complex, $\operatorname{Ar} H \mathrm{Bn}+\mathrm{Ar} H \mathrm{Bz}) ; \delta_{\mathrm{C}} 27.31,67.49$, $83.81,126.29,128.06,128.10,128.29,128.33,128.49$, 135.19, $135.91,136.21,151.89,163.04,172.09$.

Synthesis of 2i. The same procedure as described for the preparation of 2a was followed substituting Boc-Thr-OMe for Boc-Ser-OMe to give $\mathbf{2 i}$. Since attempts to crystallise the compound were unsuccessful the dehydroamino acid derivative was chromatographed through silica using diethyl ether- $n$-hexane as eluent to give $2 \mathbf{i}$ as a pure oil (87\%) (Found: $\mathrm{C}, 56.9 ; \mathrm{H}, 8.05$; $\mathrm{N}$, 4.4. Calc. for $\left.\mathrm{C}_{15} \mathrm{H}_{25} \mathrm{NO}_{6}: \mathrm{C}, 57.1 ; \mathrm{H}, 8.0 ; \mathrm{N}, 4.4 \%\right) ; \delta_{\mathrm{H}} 1.46$ $\left(18 \mathrm{H}, \mathrm{s}, \mathrm{CH}_{3} \mathrm{Boc}\right), 1.76\left(3 \mathrm{H}, \mathrm{d}, J 7.2, \gamma \mathrm{CH}_{3}\right), 3.77\left(3 \mathrm{H}, \mathrm{s}, \mathrm{CH}_{3}\right.$ OMe), $6.90(1 \mathrm{H}, \mathrm{q}, J 7.2, \beta \mathrm{C} H) ; \delta_{\mathrm{C}} 13.26,27.86,52.03,82.72$, $130.31,136.00,150.47,164.37$.

Synthesis of $\mathbf{2 j}$. The same procedure as described for the preparation of 2a was followed substituting Z-Thr-OMe for Boc-Ser-OMe to give $\mathbf{2 j}$. Since attempts to crystallise the compound were unsuccessful the dehydroamino acid derivative was chromatographed through silica using diethyl ether- $n$-hexane as eluent to give $\mathbf{2} \mathbf{j}$ as a pure oil (94\%) (Found: $\mathrm{C}, 61.6 ; \mathrm{H}, 6.8$; $\mathrm{N}, 4.0$. Calc. for $\mathrm{C}_{18} \mathrm{H}_{23} \mathrm{NO}_{6}$ : C, 61.9; $\left.\mathrm{H}, 6.6 ; \mathrm{N}, 4.0 \%\right) ; \delta_{\mathrm{H}} 1.46$ $\left(9 \mathrm{H}, \mathrm{s}, \mathrm{CH}_{3} \mathrm{Boc}\right), 1.70\left(3 \mathrm{H}, \mathrm{d}, J 7.2, \gamma \mathrm{CH}_{3}\right), 3.69\left(3 \mathrm{H}, \mathrm{s}, \mathrm{CH}_{3}\right.$ OMe), $5.21\left(2 \mathrm{H}, \mathrm{s}, \mathrm{CH}_{2} \mathrm{Z}\right), 6.95(1 \mathrm{H}, \mathrm{q}, J$ 7.2, $\beta \mathrm{CH}), 7.33$ $(5 \mathrm{H}, \mathrm{m}, \mathrm{Ar} H \mathrm{Z}) ; \delta_{\mathrm{C}} 13.27,27.77,52.08,68.25,83.27,128.19$, $127.89,128.25,128.43,129.73,135.34,137.69,150.72,151.82$, 164.06 .

Synthesis of $\mathbf{2 k}$. The same procedure as described for the preparation of 2a was followed substituting $\mathrm{Z}\left(\mathrm{NO}_{2}\right)$-Thr-OMe for Boc-Ser-OMe to give 2k $(92 \%), \mathrm{mp} 93.5-94.5^{\circ} \mathrm{C}$ (from diethyl ether-light petroleum, bp $40-60^{\circ} \mathrm{C}$ ) (Found: C, 54.7; 
$\mathrm{H}, 5.6 ; \mathrm{N}, 7.1$. Calc. for $\mathrm{C}_{18} \mathrm{H}_{22} \mathrm{~N}_{2} \mathrm{O}_{8}$ : C, 54.8; $\mathrm{H}, 5.6 ; \mathrm{N}, 7.1 \%$ ); $\delta_{\mathrm{H}} 1.47\left(9 \mathrm{H}, \mathrm{s}, \mathrm{CH}_{3} \mathrm{Boc}\right), 1.76\left(3 \mathrm{H}, \mathrm{d}, J 7.2, \gamma \mathrm{CH}_{3}\right), 3.75(3 \mathrm{H}$, s, $\left.\mathrm{CH}_{3} \mathrm{OMe}\right), 5.32\left[2 \mathrm{H}, \mathrm{d}, J 2.1, \mathrm{CH}_{2} \mathrm{Z}\left(\mathrm{NO}_{2}\right)\right], 7.01(1 \mathrm{H}, \mathrm{q}$, $J$ 7.2, $\beta \mathrm{CH}), 7.52,8.23\left[2 \mathrm{H}+2 \mathrm{H}, 2 \mathrm{~d}, J 9.0, \mathrm{Ar} H \mathrm{Z}\left(\mathrm{NO}_{2}\right)\right]$; $\delta_{\mathrm{C}} 13.37,27.77,52.26,66.75,83.73,123.73,128.09,129.63$, $137.99,142.62,147.74,149.76,151.68,163.89$.

Synthesis of 21. The same procedure as described for the preparation of $\mathbf{2 a}$ was followed substituting Tos-Thr-OMe for Boc-Ser-OMe to give $2 \mathrm{l}(87 \%), \mathrm{mp} 85-86.5^{\circ} \mathrm{C}$ (from diethyl ether- $n$-hexane) (Found: C, 55.6; H, 6.3; N, 3.9; S, 8.5. Calc. for $\left.\mathrm{C}_{17} \mathrm{H}_{23} \mathrm{NO}_{6} \mathrm{~S}: \mathrm{C}, 55.3 ; \mathrm{H}, 6.3 ; \mathrm{N}, 3.8 ; \mathrm{S}, 8.7 \%\right) ; \delta_{\mathrm{H}} 1.33(9 \mathrm{H}, \mathrm{s}$, $\mathrm{CH}_{3}$ Boc), 2.01 (3 H, d, J 7.5, $\left.\gamma \mathrm{CH}_{3}\right), 2.46\left(3 \mathrm{H}, \mathrm{s}, \mathrm{CH}_{3} \mathrm{Tos}\right)$, 3.77 ( $\left.3 \mathrm{H}, \mathrm{s}, \mathrm{CH}_{3} \mathrm{OMe}\right), 7.31$ (3 H, complex, $\left.\beta \mathrm{CH}+\mathrm{Ar} H \mathrm{Tos}\right)$, $8.01\left(2 \mathrm{H}, \mathrm{d}, J\right.$ 8.4, $\operatorname{Ar} H$ Tos); $\delta_{\mathrm{C}} 14.73,21.59,27.71,52.16$, $84.36,127.70,128.87,129.27,136.22,143.91,144.43,149.62$, 163.98 .

Synthesis of $\mathbf{2 m}$. The same procedure as described for the preparation of 2a was followed substituting $\mathrm{Z}\left(\mathrm{NO}_{2}\right)-\mathrm{Phe}(\beta$ $\mathrm{OH})-\mathrm{OMe}$ for Boc-Ser-OMe $\mathbf{2 m}$. Since attempts to crystallise the compound were unsuccessful the dehydroamino acid derivative was chromatographed by column chromatography through silica using diethyl ether- $n$-hexane as eluent to give $\mathbf{2 m}$ as a pure oil $(93 \%), \delta_{\mathrm{H}} 1.36\left(9 \mathrm{H}, \mathrm{s}, \mathrm{CH}_{3} \mathrm{Boc}\right), 3.82(3 \mathrm{H}$, s, $\left.\mathrm{CH}_{3} \mathrm{OMe}\right), 5.22\left[2 \mathrm{H}, \mathrm{q}, J 13.5, \mathrm{CH}_{2} \mathrm{Z}\left(\mathrm{NO}_{2}\right)\right], 7.35-7.46(5 \mathrm{H}$, complex, $\operatorname{Ar} H \Delta \mathrm{Phe}), 7.30\left[2 \mathrm{H}, \mathrm{d}, J\right.$ 8.7, $\left.\mathrm{ArH} \mathrm{Z}\left(\mathrm{NO}_{2}\right)\right]$, $7.63(1 \mathrm{H}, \mathrm{s}, \beta \mathrm{CH} \Delta \mathrm{Phe}), 7.08$ [2 H, d, $J$ 8.7, $\mathrm{Ar} H \mathrm{Z}\left(\mathrm{NO}_{2}\right)$ ]; $\delta_{\mathrm{C}} 27.75,52.60,66.70,83.97,123.55,124.93,126.21,128.08$, $129.02,129.66,130.40,132.38,136.99,142.35,149.49,151.34$, 164.85 .

Synthesis of 2n. The same procedure as described for the preparation of 2a was followed substituting Boc-Ser-OH for Boc-Ser-OMe and using 3.3 eq. of (Boc) $)_{2} \mathrm{O}$ to give $\mathbf{2 n}$. Since attempts to crystallise the compound were unsuccessful the dehydroamino acid derivative was chromatographed by column chromatography through silica using diethyl ether- $n$-hexane as eluent to give a pure oil that solidified on standing $(73 \%)$, mp 56-57 ${ }^{\circ} \mathrm{C}$ (Found: $\mathrm{C}, 59.5 ; \mathrm{H}, 8.3 ; \mathrm{N}, 4.1$. Calc. for $\mathrm{C}_{17} \mathrm{H}_{29} \mathrm{NO}_{6}$ : $\mathrm{C}, 59.5 ; \mathrm{H}, 8.5 ; \mathrm{N}, 4.1 \%) ; \delta_{\mathrm{H}} 1.47\left(18 \mathrm{H}, \mathrm{s}, \mathrm{CH}_{3} \mathrm{Boc}\right), 1.50(9 \mathrm{H}$, s, $\left.\mathrm{CH}_{3} \mathrm{OBu}^{t}\right), 5.57,6.27\left(1 \mathrm{H}+1 \mathrm{H}, 2 \mathrm{~s}, \beta \mathrm{CH}_{2}\right) ; \delta_{\mathrm{C}} 27.84,27.90$, $81.56,82.71,123.71,137.39,150.62,162.32$.

Synthesis of 2o. The same procedure as described for the preparation of $\mathbf{2 n}$ was followed substituting Boc-Thr-OH for Boc-Ser-OH to give 2o. Since attempts to crystallise the compound were unsuccessful the dehydroamino acid derivative was chromatographed by column chromatography through silica using diethyl ether- $n$-hexane as eluent to give $\mathbf{2 0}$ as a pure oil (73\%) (Found: $\mathrm{C}, 60.4 ; \mathrm{H}, 8.9 ; \mathrm{N}, 4.0$. Calc. for $\mathrm{C}_{18} \mathrm{H}_{31} \mathrm{NO}_{6}$ : $\mathrm{C}$, $60.5 ; \mathrm{H}, 8.7 ; \mathrm{N}, 3.9 \%) ; \delta_{\mathrm{H}} 1.46\left(18 \mathrm{H}, \mathrm{s}, \mathrm{CH}_{3} \mathrm{Boc}\right), 1.49(9 \mathrm{H}, \mathrm{s}$, $\left.\mathrm{CH}_{3} \mathrm{OBu}^{t}\right), 1.73\left(3 \mathrm{H}, \mathrm{d}, J 7.2, \gamma \mathrm{CH}_{3}\right), 6.81(1 \mathrm{H}, \mathrm{q}, J 7.2, \beta \mathrm{CH})$; $\delta_{\mathrm{C}} 13.04,27.85,27.99,80.98,82.32,131.51,135.25,150.45$, 162.84 .

Synthesis of 2a from 1p. The same procedure as described for the preparation of $\mathbf{2} \mathbf{n}$ was followed substituting H-Ser-OMe for Boc-Ser-OH to give $\mathbf{2 a}^{8}(82 \%)$.

\section{Deprotection of $\mathrm{N}$-Boc, $\mathrm{N}$-acyl dehydroamino acid esters}

Controlled potential electrolysis of $2 \mathrm{c}$. Both compartments of a two-compartment controlled-potential electrolysis cell were filled with acetonitrile containing $\mathrm{Et}_{4} \mathrm{NCl}\left(0.1 \mathrm{~mol} \mathrm{dm}^{-3}\right)$ as supporting electrolyte and $\mathrm{Et}_{3} \mathrm{NHCl}\left(0.015 \mathrm{~mol} \mathrm{dm}^{-3}\right)$ as proton donor. Then $2 \mathbf{c}(1 \mathrm{mmol})$ was added to the cathodic compartment and a cyclic voltammogram was recorded at a sweep rate of $100 \mathrm{mV} \mathrm{s}^{-1}$ in order to measure the corresponding peak potential. The potential was adjusted to a value corresponding to $50 \mathrm{mV}$ lower than the peak potential measured and the apparatus switched on. When the intensity of the current was almost zero, the reaction mixture (catholyte) was transferred to a round-bottomed flask and the solvent evaporated under reduced pressure. The residue was partitioned between $100 \mathrm{~cm}^{3}$ of diethyl ether and $50 \mathrm{~cm}^{3}$ of $\mathrm{KHSO}_{4}\left(1 \mathrm{~mol} \mathrm{dm}{ }^{-3}\right)$. The organic phase was then washed with $\mathrm{KHSO}_{4}\left(1 \mathrm{~mol} \mathrm{dm}^{-3}\right)$,

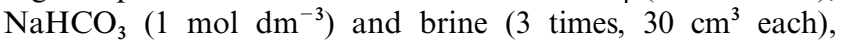
and dried over $\mathrm{MgSO}_{4}$. Concentration by evaporation under reduced pressure gave Boc- $\triangle \mathrm{Ala}-\mathrm{OMe}(88 \%)$ as a pure oil, $\delta_{\mathrm{H}} 1.49\left(9 \mathrm{H}, \mathrm{s}, \mathrm{CH}_{3}\right.$ Boc), $3.84\left(3 \mathrm{H}, \mathrm{s}, \mathrm{CH}_{3} \mathrm{OMe}\right), 5.74,6.17$ $\left(1 \mathrm{H}+1 \mathrm{H}, 2 \mathrm{~s}, \beta \mathrm{CH}_{2}\right), 7.01(1 \mathrm{H}, \mathrm{br}, \alpha \mathrm{NH}) ; \delta_{\mathrm{C}} 28.20,52.78$, $80.64,105.14,131.28,152.50,164.40$.

Controlled potential electrolysis of $2 \mathrm{e}$. The same procedure as described previously was followed substituting $2 \mathbf{e}$ for $\mathbf{2} \mathbf{c}$ to give Boc- $\Delta$ Ala-OMe $(73 \%)$.

Controlled potential electrolysis of $\mathbf{2 k}$. The same procedure as described previously was followed substituting $\mathbf{2 k}$ for $\mathbf{2 c}$ to give Boc- $\triangle \mathrm{Abu}-\mathrm{OMe}(88 \%)$ as a pure oil which solidified on standing, $\mathrm{mp}$ 69.5-71 ${ }^{\circ} \mathrm{C}$ (Found: $\mathrm{C}, 56.0 ; \mathrm{H}, 8.0 ; \mathrm{N}, 6.5$. Calc. for $\left.\mathrm{C}_{10} \mathrm{H}_{17} \mathrm{NO}_{4}: \mathrm{C}, 55.8 ; \mathrm{H}, 8.0 ; \mathrm{N}, 6.5 \%\right) ; \delta_{\mathrm{H}} 1.48\left(9 \mathrm{H}, \mathrm{s}, \mathrm{CH}_{3}\right.$ Boc), $1.81\left(3 \mathrm{H}, \mathrm{d}, J 7.2, \gamma \mathrm{CH}_{3}\right), 3.78\left(3 \mathrm{H}, \mathrm{s}, \mathrm{CH}_{3} \mathrm{OMe}\right), 5.98(1 \mathrm{H}, \mathrm{br}$, $\alpha \mathrm{N} H), 6.69(1 \mathrm{H}, \mathrm{q}, J 7.2, \beta \mathrm{CH}) ; \delta_{\mathrm{C}} 14.26,28.17,52.22,80.42$, $119.20,132.05,142.00,165.30$.

Controlled potential electrolysis of 21 . The same procedure as described previously was followed substituting $\mathbf{2 l}$ for $\mathbf{2 c}$ to give Boc- $\triangle$ Abu-OMe (78\%).

\section{Al(Hg)-mediated cleavage of $2 \mathrm{c}$}

Finely cut aluminium foil $(10 \mathrm{mmol})$ was stirred with a few drops of mercury for 30 min under a stream of nitrogen. $2 \mathbf{c}(0.5$ mmol) dissolved in ethyl ether with $1 \%$ water was then added. After $2 \mathrm{~h}$, when most of the Al had dissolved and TLC indicated only minor amounts of $\mathbf{2 c}$, more $\mathrm{Al}(\mathrm{Hg})(5 \mathrm{mmol})$ was added and left to react for a further $2 \mathrm{~h}$. The greyish solid material was then filtered off with suction and rinsed thoroughly with ethyl ether. Evaporation of the yellow filtrate gave an oil that was redissolved in chloroform and filtered to remove dark insoluble material. Evaporation of the solvent under reduced pressure gave Boc- $\Delta \mathrm{Ala}-\mathrm{OMe}(87 \%)$ as a pure oil.

\section{Al(Hg)-mediated cleavage of $2 \mathbf{k}$}

The same procedure as described above was followed substituting $\mathbf{2 k}$ for $\mathbf{2} \mathbf{c}$ to give Boc- $\triangle \mathrm{Abu}-\mathrm{OMe}(95 \%)$.

\section{Acidolysis of $2 \mathrm{j}$}

To $2 \mathbf{j}$ ( $1 \mathrm{mmol}) 3 \mathrm{~cm}^{3}$ of TFA were added and the solution left to stand for $1 \mathrm{~h}$. Excess TFA was removed by evaporation under reduced pressure to give $\mathrm{Z}-\Delta \mathrm{Abu}-\mathrm{OMe}^{20}(87 \%)$, mp $69.5-$ $70.5^{\circ} \mathrm{C}$ (from diethyl ether- $n$-hexane) (Found: $\mathrm{C}, 62.6 ; \mathrm{H}, 6.3$; $\mathrm{N}$, 5.6. Calc. for $\left.\mathrm{C}_{13} \mathrm{H}_{15} \mathrm{NO}_{4}: \mathrm{C}, 62.6 ; \mathrm{H}, 6.1 ; \mathrm{N}, 5.6 \%\right) ; \delta_{\mathrm{H}} 1.83$ $\left(3 \mathrm{H}, \mathrm{d}, J 7.2, \gamma \mathrm{CH}_{3}\right), 3.77\left(3 \mathrm{H}, \mathrm{s}, \mathrm{CH}_{3} \mathrm{OMe}\right), 5.16\left(2 \mathrm{H}, \mathrm{s}, \mathrm{CH}_{2}\right.$ $\mathrm{Z}), 6.77(1 \mathrm{H}, \mathrm{q}, J 7.2, \beta \mathrm{CH}), 7.36(5 \mathrm{H}, \mathrm{m}, \operatorname{Ar} H \mathrm{Z}) ; \delta_{\mathrm{C}} 14.26$, $52.30,67.33,128.12,128.22,128.52,133.27,136.01,164.98$.

\section{Acidolysis of 2k}

The same procedure as described previously was followed substituting $2 \mathbf{k}$ for $\mathbf{2} \mathbf{j}$ to give $\mathrm{Z}\left(\mathrm{NO}_{2}\right)-\triangle \mathrm{Abu}-\mathrm{OMe}(85 \%)$, mp 120 $121{ }^{\circ} \mathrm{C}$ (from diethyl ether) (Found: $\mathrm{C}, 53.0 ; \mathrm{H}, 4.9 ; \mathrm{N}, 9.2$. Calc. for $\left.\mathrm{C}_{13} \mathrm{H}_{14} \mathrm{~N}_{2} \mathrm{O}_{6}: \mathrm{C}, 53.1 ; \mathrm{H}, 4.8 ; \mathrm{N}, 9.5 \%\right) ; \delta_{\mathrm{H}} 1.83(3 \mathrm{H}, \mathrm{d}, J 7.2$, $\left.\gamma \mathrm{CH}_{3}\right), 3.79\left(3 \mathrm{H}, \mathrm{s}, \mathrm{CH}_{3} \mathrm{OMe}\right), 5.26\left[2 \mathrm{H}, \mathrm{s}, \mathrm{CH}_{2} \mathrm{Z}\left(\mathrm{NO}_{2}\right)\right], 6.30$ $(1 \mathrm{H}, \mathrm{br}, \alpha \mathrm{NH}), 6.82(1 \mathrm{H}, \mathrm{q}, J$ 7.2, $\beta \mathrm{CH}), 7.54,8.24[2 \mathrm{H}+$ $2 \mathrm{H}, 2 \mathrm{~d}, J$ 8.7, $\left.\mathrm{Ar} H \mathrm{Z}\left(\mathrm{NO}_{2}\right)\right]$; $\delta_{\mathrm{C}} 14.26,52.41,65.72,123.74$, $127.99,128.11,133.93,143.42,164.84$. 


\section{Saponification of Z-Abu-OMe}

To Z- $\Delta$ Abu-OMe $(1 \mathrm{mmol})$ in dioxane $\left(0.2 \mathrm{~mol} \mathrm{dm}^{-3}\right), 3 \mathrm{~cm}^{3}$ of $\mathrm{NaOH}\left(1 \mathrm{~mol} \mathrm{dm}{ }^{-3}\right)$ were added. The solution was stirred at room temperature for $2 \mathrm{~h}$, acidified to $\mathrm{pH}$ 2-3 with $\mathrm{KHSO}_{4}$ $\left(1 \mathrm{~mol} \mathrm{dm}^{-3}\right)$ and then extracted with ethyl acetate. The organic phase was dried over $\mathrm{MgSO}_{4}$ and evaporated under reduced pressure to give $\mathrm{Z}-\Delta \mathrm{Abu}-\mathrm{OH}(77 \%), \mathrm{mp} 174-176{ }^{\circ} \mathrm{C}$ (from ethyl acetate-diethyl ether) (Found: $\mathrm{C}, 61.1 ; \mathrm{H}, 5.6 ; \mathrm{N}, 6.0$. Calc. for $\mathrm{C}_{12} \mathrm{H}_{13} \mathrm{NO}_{4}$ : C, 61.3; $\left.\mathrm{H}, 5.6 ; \mathrm{N}, 5.95 \%\right) ; \delta_{\mathrm{H}}[300 \mathrm{MHz}$; $\left.\left(\mathrm{CD}_{3}\right)_{2} \mathrm{SO}\right] 1.66\left(3 \mathrm{H}, \mathrm{d}, J 6.9, \gamma \mathrm{CH}_{3}\right), 5.03\left(2 \mathrm{H}, \mathrm{s}, \mathrm{CH} \mathrm{Z}_{2} \mathrm{Z}\right), 6.47$ $(1 \mathrm{H}, \mathrm{q}, J 6.9, \beta \mathrm{CH}), 7.35(5 \mathrm{H}, \mathrm{m}, \mathrm{Ar} H \mathrm{Z}), 8.61(1 \mathrm{H}, \mathrm{br}, \alpha \mathrm{NH})$; $\delta_{\mathrm{C}}\left[75.4 \mathrm{MHz} ;\left(\mathrm{CD}_{3}\right)_{2} \mathrm{SO}\right] 13.17,65.63,127.62,128.32,128.76$, 131.36, 136.79, 154.24, 165.62.

\section{Synthesis of Z- $\triangle \mathrm{Abu}-\mathrm{Gly}-\mathrm{OMe}$}

$\mathrm{Z}-\Delta \mathrm{Abu}-\mathrm{OH}$ was reacted with $\mathrm{H}-\mathrm{Gly}-\mathrm{OMe} \cdot \mathrm{HCl}$ in ethyl acetate using the standard DCC-HOBt procedure to give Z- $\triangle \mathrm{Abu}-\mathrm{Gly}-$ OMe $(85 \%), \mathrm{mp} 90-90.5^{\circ} \mathrm{C}$ (from diethyl ether- $n$-hexane) (Found: C, 58.7; $\mathrm{H}, 6.1 ; \mathrm{N}, 9.1$. Calc. for $\mathrm{C}_{15} \mathrm{H}_{18} \mathrm{~N}_{2} \mathrm{O}_{5}$ : C, 58.8; $\mathrm{H}, 5.9 ; \mathrm{N}, 9.1 \%) ; \delta_{\mathrm{H}} 1.77\left(3 \mathrm{H}, \mathrm{d}, J 6.9, \gamma \mathrm{CH}_{3}\right), 3.76\left(3 \mathrm{H}, \mathrm{s}, \mathrm{CH}_{3}\right.$ $\mathrm{OMe}), 4.07\left(2 \mathrm{H}, \mathrm{d}, J 4.5, \mathrm{CH}_{2} \mathrm{Gly}\right), 5.16\left(2 \mathrm{H}, \mathrm{s}, \mathrm{CH}_{2} \mathrm{Z}\right), 6.20$ ( $1 \mathrm{H}, \mathrm{br}, \alpha \mathrm{N} H \mathrm{Gly}), 6.56$ (1 H, q, $J$ 6.9, $\beta \mathrm{CH} \Delta \mathrm{Abu}), 6.61(1 \mathrm{H}$, br, $\alpha \mathrm{NH} \Delta \mathrm{Abu}), 7.36(5 \mathrm{H}, \mathrm{s}, \operatorname{Ar} H \mathrm{Z}) ; \delta_{\mathrm{C}} 13.37,41.13,52.38$, $67.55,98.57,128.17,128.32,128.55,129.28,135.81,164.80$, 170.25 .

\section{Preparation of $\mathbf{N}$-acyl dipeptides esters 3a-3f}

In all cases the $N$-acyl protected amino acid was reacted with the appropriate amino acid ester in ethyl acetate using the standard DCC-HOBt procedure to give: 3a (85\%), mp 130 $131.5^{\circ} \mathrm{C}$ (from ethyl acetate- $n$-hexane) (Found: $\mathrm{C}, 57.3 ; \mathrm{H}, 5.2$; $\mathrm{N}, 9.1$. Calc. for $\left.\mathrm{C}_{22} \mathrm{H}_{25} \mathrm{~N}_{3} \mathrm{O}_{8}: \mathrm{C}, 57.5 ; \mathrm{H}, 5.5 ; \mathrm{N}, 9.15 \%\right) ; \delta_{\mathrm{H}} 1.26$ ( $3 \mathrm{H}, \mathrm{t}, J$ 7.2, $\mathrm{CH}_{3} \mathrm{OEt}$ ), 3.12 ( $\left.2 \mathrm{H}, \mathrm{m}, \beta \mathrm{CH}_{2} \mathrm{Phe}\right), 3.55(2 \mathrm{H}, \mathrm{m}$, $\mathrm{BCH}_{2}$ Ser $), 4.00(1 \mathrm{H}, \mathrm{m}, \alpha \mathrm{CH}$ Ser $), 4.19\left(2 \mathrm{H}, \mathrm{q}, J\right.$ 7.2, $\mathrm{CH}_{2}$ OEt), 4.84 (1 H, q, $J$ 6.6, $\alpha \mathrm{CH}$ Phe), 5.18 [2 H, s, $\mathrm{CH}_{2} \mathrm{Z}\left(\mathrm{NO}_{2}\right)$ ], $5.92(1 \mathrm{H}, \mathrm{d}, J 6.6, \alpha \mathrm{N} H$ Phe), $6.93(1 \mathrm{H}, \mathrm{d}, J 7.5, \alpha \mathrm{N} H$ Ser $)$, 7.26 (5 H, m, ArH Phe), 7.49, 8.21 [2 H $+2 \mathrm{H}, 2 \mathrm{~d}, J$ 8.7, ArH $\left.\mathrm{Z}\left(\mathrm{NO}_{2}\right)\right] ; \delta_{\mathrm{C}} 14.04,37.50,49.14,55.52,61.86,62.79,65.56$, $123.75,127.18,128.01,128.55,129.07,135.60,143.46,147.61$, $155.78,156.86,171.49 ; 3 \mathbf{b}(81 \%)$, oil (lit., ${ }^{33}$ oil), $\delta_{\mathrm{H}} 1.41(3 \mathrm{H}$, $J$ 6.9, $\mathrm{BCH}_{3}$ Ala), 1.46 ( $9 \mathrm{H}, \mathrm{s}, \mathrm{CH}_{3}$ Boc $), 3.80\left(3 \mathrm{H}, \mathrm{s}, \mathrm{CH}_{3}\right.$ $\mathrm{OMe}), 4.04$ ( $\left.2 \mathrm{H}, \mathrm{m}, \mathrm{\beta CH}_{2} \mathrm{Ser}\right), 4.16(1 \mathrm{H}, \mathrm{m}, \alpha \mathrm{CH}$ Ser $), 4.63$ (1 H, m, $\alpha \mathrm{CH}$ Ala $), 5.03(1 \mathrm{H}, \mathrm{d}, J 6.0, \alpha \mathrm{NH}$ Ala $), 7.11(1 \mathrm{H}$, $\mathrm{d}, J$ 6.6, $\alpha \mathrm{NH}$ Ser); 3c (71\%), mp $105.5-107^{\circ} \mathrm{C}$ (from ethyl acetate-petroleum ether) (lit. $\left.{ }^{34} 105-106{ }^{\circ} \mathrm{C}\right) ; 3 \mathbf{3 d}(92 \%), \mathrm{mp} 94$ $95^{\circ} \mathrm{C}$ (from ethyl acetate- $n$-hexane) (lit., ${ }^{35} 104-105^{\circ} \mathrm{C}$ ); $3 \mathbf{e}^{36}$ (92\%), oil, $\delta_{\mathrm{H}} 1.24\left(3 \mathrm{H}, \mathrm{d}, J 6.3, \gamma \mathrm{CH}_{3} \mathrm{Thr}\right), 1.46\left(9 \mathrm{H}, \mathrm{s}, \mathrm{CH}_{3}\right.$ Boc), 3.80 (3 H, s, $\left.\mathrm{CH}_{3} \mathrm{OMe}\right), 3.96\left(2 \mathrm{H}, \mathrm{m}, \beta \mathrm{CH}_{2} \mathrm{Ser}\right), 4.14$ (1 H, m, $\beta \mathrm{CH}$ Thr $), 4.36(1 \mathrm{H}, \mathrm{m}, \alpha \mathrm{CH}$ Ser $), 4.66(1 \mathrm{H}, \mathrm{m}, \alpha \mathrm{CH}$ Thr $)$, 5.61, $7.40(1 \mathrm{H}+1 \mathrm{H}$, br, $\alpha \mathrm{N} H$ Ser and $\alpha \mathrm{N} H \mathrm{Thr})$; $\delta_{\mathrm{C}} 18.49,28.23,52.80,55.72,58.88,62.38,67.29,80.47,156.00$ $170.74,171.43 ; 3 f(76 \%), \mathrm{mp} 121-123^{\circ} \mathrm{C}$ (from ethyl acetatediethyl ether) (Found: $\mathrm{C}, 50.3 ; \mathrm{H}, 7.6 ; \mathrm{N}, 8.6$. Calc. for $\left.\mathrm{C}_{14} \mathrm{H}_{26} \mathrm{~N}_{2} \mathrm{O}_{7}: \mathrm{C}, 50.3 ; \mathrm{H}, 7.8 ; \mathrm{N}, 8.4 \%\right) ; \delta_{\mathrm{H}} 1.23(6 \mathrm{H}, \mathrm{d}, J 6.3$, $\left.\gamma \mathrm{CH}_{3} \mathrm{Thr}\right), 1.46$ (9 H, s, $\left.\mathrm{CH}_{3} \mathrm{Boc}\right), 3.79\left(3 \mathrm{H}, \mathrm{s}, \mathrm{CH}_{3} \mathrm{OMe}\right), 4.15$ $(1 \mathrm{H}, \mathrm{m}, \alpha \mathrm{CH} \mathrm{Thr}), 4.14-3.37(2 \mathrm{H}$, complex, $\beta \mathrm{CH} \mathrm{Thr}+\beta \mathrm{CH}$ Thr), $4.60(1 \mathrm{H}, \mathrm{m}, \alpha \mathrm{CH} \mathrm{Thr}), 5.61,7.31(1 \mathrm{H}+1 \mathrm{H}, 2 \mathrm{~d}, J 7.5$, $J$ 9.0, $\alpha \mathrm{N} H$ Thr and $\alpha \mathrm{N} H \mathrm{Thr}) ; \delta_{\mathrm{C}} 18.19,19.98,28.29,52.71$, $57.49,58.07,67.06,67.96,80.55,156.38,171.21,172.08$.

\section{Synthesis of dehydroamino acid containing dipeptides}

Synthesis of 4a. To a solution of 3a in dry acetonitrile (1 mol $\mathrm{dm}^{-3}$ ) 0.3 eq. of DMAP was added followed by 3.3 eq. of tertbutyl pyrocarbonate under rapid stirring at room temperature. The reaction was stirred for $12 \mathrm{~h}$ while monitored by TLC (diethyl ether- $n$-hexane, 2:1). Evaporation under reduced pressure gave a residue that was partitioned between $200 \mathrm{~cm}^{3}$ of diethyl ether and $100 \mathrm{~cm}^{3}$ of $\mathrm{KHSO}_{4}\left(1 \mathrm{~mol} \mathrm{dm}{ }^{-3}\right)$. The organic phase was thoroughly washed with $\mathrm{KHSO}_{4}\left(1 \mathrm{~mol} \mathrm{dm}{ }^{-3}\right)$, $\mathrm{NaHCO}_{3}\left(1 \mathrm{~mol} \mathrm{dm}^{-3}\right)$ and saturated brine $\left(3 \times 50 \mathrm{~cm}^{3}\right.$ each $)$, and dried over $\mathrm{MgSO}_{4}$. Removal of the solvent afforded 4a. Since attempts to crystallise the compound were unsuccessful the dehydroamino acid derivative was chromatographed by column chromatography through silica using diethyl ether- $n$ hexane as eluent to give $4 \mathbf{a}$ as a pure oil $(93 \%), \delta_{\mathrm{H}} 1.27(3 \mathrm{H}, \mathrm{t}$, $J$ 7.1, $\left.\mathrm{CH}_{3} \mathrm{OEt}\right), 1.50,1.57\left(9 \mathrm{H}+9 \mathrm{H}, 2 \mathrm{~s}, \mathrm{CH}_{3} \mathrm{Boc}+\mathrm{CH}_{3}\right.$ Boc), 3.51 ( $\left.2 \mathrm{H}, \mathrm{m}, \mathrm{BCH}_{2} \mathrm{Phe}\right), 4.30\left(2 \mathrm{H}, \mathrm{q}, J 7.1, \mathrm{CH}_{2} \mathrm{OEt}\right)$, $5.05(1 \mathrm{H}, \mathrm{dd}, J 6.5, J 10.1, \alpha \mathrm{CH} \mathrm{Phe}), 5.20\left[2 \mathrm{H}, \mathrm{s}, \mathrm{CH}_{2}\right.$ $\left.\mathrm{Z}\left(\mathrm{NO}_{2}\right)\right], 5.75,5.95\left(1 \mathrm{H}+1 \mathrm{H}, 2 \mathrm{~s}, \beta \mathrm{CH}_{2} \Delta \mathrm{Ala}\right), 7.20(5 \mathrm{H}, \mathrm{m}$, $\mathrm{Ar} H$ Phe), 7.55, $8.25\left[2 \mathrm{H}+2 \mathrm{H}, 2 \mathrm{~d}, J\right.$ 8.6, $\left.\mathrm{ArH} \mathrm{Z}\left(\mathrm{NO}_{2}\right)\right]$; $\delta_{\mathrm{C}} 13.99,27.62,27.78,33.83,48.93,54.23,62.20,66.92,82.90$, $85.40,105.42,123.67,126.93,128.18,128.60,128.66,131.42$, $136.18,142.95,147.57,149.44,152.99,160.31,167.75$.

Synthesis of $\mathbf{4 b}$. The same procedure as described for the preparation of $\mathbf{4 a}$ was followed substituting $\mathbf{3 b}$ for $\mathbf{3 a}$ to give 4b (91\%), mp $102.5-103.5^{\circ} \mathrm{C}$ (from diethyl ether- $n$-hexane) (Found: C, 56.3; $\mathrm{H}, 7.7 ; \mathrm{N}, 6.1$. Calc. for $\mathrm{C}_{22} \mathrm{H}_{36} \mathrm{~N}_{2} \mathrm{O}_{9}$ : C, 55.9; $\mathrm{H}, 7.7 ; \mathrm{N}, 5.9 \%) ; \delta_{\mathrm{H}} 1.43\left(3 \mathrm{H}, \mathrm{d}, J 6.9, \beta C \mathrm{H}_{3} \mathrm{Ala}\right), 1.48(27 \mathrm{H}, \mathrm{s}$, $\left.\mathrm{CH}_{3} \mathrm{Boc}\right), 3.76\left(3 \mathrm{H}, \mathrm{s}, \mathrm{CH}_{3} \mathrm{OMe}\right), 5.37(1 \mathrm{H}, \mathrm{q}, J$ 6.9, $\alpha \mathrm{CH}$ Ala), 5.77, $6.35\left(1 \mathrm{H}+1 \mathrm{H}, 2 \mathrm{~s}, \beta \mathrm{CH}_{2} \Delta \mathrm{Ala}\right) ; \delta_{\mathrm{C}} 14.97,27.79$, 27.96, 52.33, 56.44, 82.94, 83.37, 124.82, 135.89, 150.87, 152.00, $163.97,173.75$

Synthesis of $\mathbf{4 c}$. The same procedure as described for the preparation of $\mathbf{4 a}$ was followed substituting $\mathbf{3 c}$ for $\mathbf{3 a}$ to give $\mathbf{4 c}$. Since attempts to crystallise the compound were unsuccessful the dehydroamino acid derivative was chromatographed by column chromatography through silica using diethyl ether- $n$ hexane as eluent to give $\mathbf{4 c}$ as a pure oil $(81 \%), \delta_{\mathrm{H}} 1.48,1.50$, $1.60\left(9 \mathrm{H}+9 \mathrm{H}+9 \mathrm{H}, 3 \mathrm{~s}, \mathrm{CH}_{3} \mathrm{Boc}+\mathrm{CH}_{3} \mathrm{Boc}+\mathrm{CH}_{3} \mathrm{Boc}\right)$, $2.31\left(3 \mathrm{H}, \mathrm{d}, J 8.1, \gamma \mathrm{CH}_{3} \Delta \mathrm{Abu}\right), 3.78\left(3 \mathrm{H}, \mathrm{s}, \mathrm{CH}_{3} \mathrm{OMe}\right), 4.34$ $\left(2 \mathrm{H}, \mathrm{s}, \mathrm{CH}_{2} \mathrm{Gly}\right), 5.1\left(2 \mathrm{H}, \mathrm{s}, \mathrm{CH}_{2} \mathrm{Z}\right), 7.16(1 \mathrm{H}, \mathrm{q}, J$ 8.1, $\beta \mathrm{CH}$ $\Delta \mathrm{Abu}), 7.40-7.33(5 \mathrm{H}, \mathrm{m}, \operatorname{Ar} H \mathrm{Z}) ; \delta_{\mathrm{C}} 13.03,27.91,39.08$, $52.71,67.55,85.05,125.48,126.22,148.35,149.31,160.55$, 166.96.

Synthesis of 4d. The same procedure as described for the preparation of $4 \mathbf{a}$ was followed substituting $3 \mathbf{d}$ for $\mathbf{3 a}$ to give $\mathbf{4 d}$ (84\%), mp $111.5-112.5^{\circ} \mathrm{C}$ (from diethyl ether-light petroleum, bp 40-60 ${ }^{\circ} \mathrm{C}$ ) (Found: C, 56.7; H, 7.9; N, 5.8. Calc. for $\mathrm{C}_{23} \mathrm{H}_{38} \mathrm{~N}_{2} \mathrm{O}_{9}$ : C, 56.8; H, 7.9; N, 5.8\%); $\delta_{\mathrm{H}} 1.45(3 \mathrm{H}, \mathrm{d}, J$ 6.1, $\beta_{\mathrm{C} \mathrm{H}_{3}}$ Ala), $1.51\left(27 \mathrm{H}, \mathrm{s}, \mathrm{CH}_{3} \mathrm{Boc}\right), 1.76\left(3 \mathrm{H}, \mathrm{d}, J \mathrm{~J} .3, \gamma \mathrm{CH}_{3}\right.$ $\Delta \mathrm{Abu}), 3.75\left(3 \mathrm{H}, \mathrm{s}, \mathrm{CH}_{3} \mathrm{OMe}\right), 5.22(1 \mathrm{H}, \mathrm{q}, J 6.1, \alpha \mathrm{CH}$ Ala), $6.94(1 \mathrm{H}, \mathrm{q}, J 7.3, \beta \mathrm{CH} \Delta \mathrm{Abu}) ; \delta_{\mathrm{C}} 14.43,18.08,28.26$, $33.88,52.25,83.09,125.87,134.40,150.81,154.38,164.73$, 171.00 .

Synthesis of 4e. The same procedure as described for the preparation of $\mathbf{4 a}$ was followed substituting $\mathbf{3 e}$ for $\mathbf{3} \mathbf{a}$ and using 4.4 eq. of tert-butyl pyrocarbonate to give $4 \mathrm{e}(83 \%)$, mp $118.5-$ $119.5^{\circ} \mathrm{C}$ (from diethyl ether-light petroleum, bp $40-60^{\circ} \mathrm{C}$ ) (Found: C, 57.1; H, 7.7; N, 5.8. Calc. for $\mathrm{C}_{23} \mathrm{H}_{36} \mathrm{~N}_{2} \mathrm{O}_{9}$ : C, 57.0; $\mathrm{H}, 7.5 ; \mathrm{N}, 5.8 \%) ; \delta_{\mathrm{H}} 1.46\left[18 \mathrm{H}, \mathrm{s}, \mathrm{CH}_{3}(\mathrm{Boc})_{2}\right], 1.49\left(9 \mathrm{H}, \mathrm{s}, \mathrm{CH}_{3}\right.$ Boc), $1.75\left(3 \mathrm{H}, \mathrm{d}, J\right.$ 7.2, $\left.\gamma \mathrm{CH}_{3} \Delta \mathrm{Abu}\right), 3.80\left(3 \mathrm{H}, \mathrm{s}, \mathrm{CH}_{3} \mathrm{OMe}\right)$, $6.17,6.18\left(1 \mathrm{H}+1 \mathrm{H}, 2 \mathrm{~d}, J 0.9, \beta \mathrm{CH} H_{2} \Delta \mathrm{Ala}\right), 6.80(1 \mathrm{H}, \mathrm{q}$, $J 7.2, \beta \mathrm{CH} \Delta \mathrm{Abu}) ; \delta_{\mathrm{C}} 14.45,27.80,27.83,52.38,83.36,123.49$, $132.62,134.78,138.78,150.16,163.78,166.70$.

Synthesis of $4 \mathbf{f}$. The same procedure as described for the preparation of $\mathbf{4 e}$ was followed substituting $3 \mathbf{f}$ for $\mathbf{3 e}$ to give 4f. Since attempts to crystallise the compound were unsuccessful the dehydroamino acid derivative was chromatographed by column chromatography through silica using diethyl ether- $n$ hexane as eluent to give a pure oil $(74 \%), \delta_{\mathrm{H}} 1.46\left[18 \mathrm{H}, \mathrm{s}, \mathrm{CH}_{3}\right.$ (Boc) $)_{2}$ ], 1.49 (9 H, s, $\mathrm{CH}_{3}$ Boc), 1.73, $1.76(3 \mathrm{H}+3 \mathrm{H}, 2 \mathrm{~d}, J 7.0$, $\left.\gamma \mathrm{CH}_{3} \Delta \mathrm{Abu}\right), 3.75\left(3 \mathrm{H}, \mathrm{s}, \mathrm{CH}_{3} \mathrm{OMe}\right), 6.66,6.88(1 \mathrm{H}+1 \mathrm{H}$, $2 \mathrm{q}, J 7.0, \beta \mathrm{CH} \Delta \mathrm{Abu}) ; \delta_{\mathrm{C}} 13.48,14.29,27.72,27.78,52.05$, 
$83.01,83.25,129.09,133.10,136.89,138.04,149.89,151.08$, $164.00,166.64$.

\section{References}

1 U. Schmidt, J. Hausler, E. Olher and H. Poisel, Fortschr. Chem. Org. Naturst., 1977, 37, 251.

2 K. Noda, Y. Shimohigashi and N. Izumiya, The Peptides, Analysis, Synthesis, Biology, Academic Press, New York, 1983, vol. 5, p. 286.

3 U. Schmidt, A. Lieberknecht and J. Wild, Synthesis, 1988, 159.

4 G. Jung, Angew. Chem., Int. Ed. Engl., 1991, 30, 1051.

5 S. Nomoto, A. Sano and T. Shiba, Tetrahedron Lett., 1979, 6, 521.

6 R. H. Mazur and D. R. Pilipauskas, Peptides 1982, Walter de Gruyter, Berlin, 1983, p. 319.

7 M. Barbaste, V. Rolland-Fulcrand, M. L. Roumestant, P. Viallefont and J. Martinez, Tetrahedron Lett., 1998, 39, 6287.

8 P. M. T. Ferreira, H. L. S. Maia and L. S. Monteiro, Tetrahedron Lett., 1999, 40, 4099.

9 V. E. Price and J. P. Greenstein, J. Biol. Chem., 1947, 171, 477.

10 F. Wieland, G. Ohnacker and W. Ziegler, Chem. Ber., 1957, 90, 194.

11 M. J. Miller, J. Org. Chem., 1980, 45, 3131.

12 Y. Nakagawa, T. Tsuno, K. Nakajima, M. Iwai, H. Kawai and K. Okawa, Bull. Chem. Soc. Jpn., 1972, 45, 1162.

13 T. L. Sommerfeld and D. Seebach, Helv. Chim. Acta, 1993, 76, 1702.

14 D. B. Berkowitz and M. L. Pederson, J. Org. Chem., 1194, 5476.

15 P. M. T. Ferreira, H. L. S. Maia and L. M. Rodrigues, Peptides 1996, Mayflower Scientific Ltd., Kingswinford, 1998, p. 377.

16 L. Grehn, K. Gunnarsson, H. L. S. Maia, M. I. Montenegro, L. Pedro and U. Ragnarsson, J. Chem. Res. (S), 1988, 399; J. Chem. Res. (M), 1988, 3081 .

17 L. Grehn, H. L. S. Maia, L. S. Monteiro, M. I. Montenegro and U. Ragnarsson, J. Chem. Res. (S), 1991, 144; J. Chem. Res. (M), 1991, 1501.

18 L. Grehn, K. Gunnarsson and U. Ragnarsson, Acta Chem. Scand., Ser. $B, 1986, \mathbf{4 0}, 745$.
19 P. M. T. Ferreira, H. L. S. Maia and L. S. Monteiro, Tetrahedron Lett., 1998, 39, 9575.

20 A. Srinivasan, R. W. Stephesen and R. K. Olsen, J. Org. Chem., 1977, 42, 2256.

21 W. A. Nugent, PCT Int. Appl. W09616021 (Cl. C07C231/14), US Appl. 340,781,17, Chem. Abstr., 1996, 143302k.

22 K. Takeda, A. Akiyama, H. Nakamura, S. Takizawa, Y. Mizuno, H. Takayanagi and Y. Harigaya, Synthesis, 1994, 1063.

23 B. Nyassé, L. Grehn, H. L. S. Maia, L. S. Monteiro and U. Ragnarsson, J. Org. Chem., 1999, 64, 7135.

24 C. Shin, Y. Yonezawa, M. Takahashi and J. Yoshimura, Bull. Chem. Soc. Jpn., 1981, 54, 1132.

25 B. Iselin and R. Schwayer, Helv. Chim. Acta, 1961, 44, 169

26 C. H. Hassall and J. O. Thomas, J. Chem. Soc., 1968, 1495.

27 A. Stoll and T. Petrzilka, Helv. Chim. Acta, 1952, 35, 589.

28 E. M. Fry, J. Org. Chem., 1950, 15, 438.

29 M. Murata, M. Tsutsumi, K. Matsuda, K. Mattori and T. Nakajima, Eur. Pat. Appl., EP 272,455, Chem. Abstr., 1988, 109, $210794 \mathrm{~g}$.

30 I. Yamashina, H. Sugimoto and I. Makino, Jpn. Kokai Tokkyo Koho, JP 03,176,497 [91,176,497], Chem. Abstr., 1992, 116, 84178p.

31 V. F. Podgornova, N. N. Zentsova, N. K. Aukone and U. O. Kalei, Khim. Prir. Soedin., 1979, 4, 543.

32 G. Kotlanz, Ann. Acad. Med. Gedanensis, 1988, 18, 193.

33 L. Sannama, T. Kanmera, H. Aoyagi and N. Izumiya, Int. J. Pept. Protein Res., 1979, 13, 207.

34 E. Schaich and F. Schneider, Hoppe-Seyler's Z. Physiol. Chem., 1974, 355, 952

35 S. K. Gupta, Synthetic Peptides, Van Nostrand Reinhold, New York, 1971, vol. 2, p. 40.

36 D. F. Veber, F. W. Holly, R. G. Strachan, W. J. Palaveda and R. Nutt, Ger. Offen. 2,635,558 (Cl. C07C103/52); US Appl. 603,067, Chem. Abstr., 1977, 87, 23741c.

Paper 9/04730A 